\title{
FAILURE OF GYMNEMA EXTRACT TO INHIBIT THE SUGAR RECEPTORS OF TWO INVERTEBRATES
}

\author{
JAMES L. LARIMER* and BRUCE OAKLEY† \\ Department of Zoology, University of Texas, Austin, and \\ Department of Zoology, The University of Michigan, Ann Arbor
}

(Received 4 December 1967)

\begin{abstract}
Tests have been made to determine if the inhibitor of sweet reception in man, gymnemic acid, would affect the responses of two invertebrates, the crayfish, Procambarus clarkii and fly, Sarcophaga sp.

2. In behavioral tests involving both these animals, as well as electrophysiological recordings from chemoreceptive hairs of flies, Gymnema extract produced no measurable inhibition of sugar chemoreception.

3. Given the high degree of structural and conformational specificity of the crayfish and fly sugar receptors, as compared to those of man, competitive inhibition by Gymnema may not be sufficiently strong to overcome the binding of sugars to the invertebrate receptor membranes.
\end{abstract}

\section{INTRODUCTION}

ONE OF the few specific inhibitors of chemoreceptors is a chemical obtained from the tropical plant, Gymnema sylvestre. Extracts made from the leaves of this plant reversibly block the sugar or sweet receptors in man, without modification of those mediating the tastes of sour or salty substances (Warren \& Pfaffmann, 1959; Diamant et al., 1965). Although some invertebrate sugar receptors are now well known (Hodgson, 1957; Dethier, 1963; Ashby \& Larimer, 1965) their behavior toward Gymnema extract has not been examined. In this paper we report the results of a series of tests made on the sugar receptors of the crayfish Procambarus clarkii and on the flesh fly, Sarcophaga sp. when the animals were treated in various ways with Gymnema extract.

\section{Crayfish}

\section{METHODS}

The extract was made by heating leaves of the plant Gymnema sylvestre in water at $80^{\circ} \mathrm{C}$ for $4 \mathrm{hr}$ according to the method of Diamant et al. (1965). The final solution, after filtration, was greenish-orange in color and represented $0.47 \mathrm{~g}$ leaves $/ \mathrm{ml}$ of solution. This corresponds to slightly more than $2 \%$ gymnemic acid

* Research supported in part by NIH Grant NB-05423 from the National Institute of Neurological Diseases and Blindness.

$\dagger$ Research supported in part by NSF -Institutional Grant No. 109 and by NIH Grant NB-07072 from the National Institute of Neurological Diseases and Blindness. 
according to the data of Warren \& Pfaffmann (1959). Tests made on three human subjects showed that it was effective in raising the detection threshold for sucrose from a value of about $0.02 \mathrm{M}$ to between 0.2 and $0.4 \mathrm{M}$. When not in use, the extract was refrigerated.

The experiments designed to test the effects of Gymnema extract on crayfish sugar reception followed the methods previously used (Larimer, 1964; Ashby \& Larimer, 1965). The behavioral response to sugars involved an inhibition of the heart that was recorded by EKG electrodes. The sugar chosen was glucose because it is almost 100 per cent effective in evoking a complete heart block. The standard dose was $2.5 \mathrm{ml}$ of $0.25 \mathrm{M}$ glucose introduced slowly into the ventilation stream. Controls consisted of either water or $0.25 \mathrm{M}$ sucrose, neither of which stimulate. The animals were restrained on a platform in a water bath of 9.18 1. The platform and animal, or the animal alone was completely immersed in Gymnema extract for periods of 20-30 min. The animal was then thoroughly rinsed in water for about $1 \mathrm{~min}$ and then given in sequence the $0.25 \mathrm{M}$ glucose, $0.25 \mathrm{M}$ sucrose and occasionally, water. The bath was changed frequently to prevent accumulation of the stimulant. Twenty-one crayfish were used in these experiments. Thirty-seven control observations were made and compared with forty observations after treatment with Gymnema extract.

\section{Electrophysiological methods using fies}

Action potentials were recorded from the neurons in individual taste hairs on the fly labellum by using the method of Hodgson et al. (1955). This involves placing over the tip of the hair a glass pipette that contains the stimulating chemical and enough salt to conduct action potentials to a preamplifier via a silver wire in the pipette. A second silver wire in the crushed head served as the reference electrode. The impulse activity was photographed from an oscilloscope trace with a kymograph camera. Gymnema extract was made by the method outlined above; $0.1 \%, 1 \cdot 5 \%$, and $10 \%$ solutions were applied to the taste hair by: (a) touching a droplet to the tip of a sugar-sensitive hair for at least $1 \mathrm{~min}$, or (b) saturating the entire labellum with Gymnema extract for at least $1 \mathrm{~min}$, or (c) putting the Gymnema extract in the stimulating-recording pipette prior to any contact with the hair. The most common procedure was to place the following mixture in the pipette before any attempt at recording: $1 \%$ Gymnema extract, $0.5 \mathrm{M}$ sucrose, and either $0.01 \mathrm{M} \mathrm{NaCl}$ or $0.05 \mathrm{M} \mathrm{NaCl}$. Then, one could look for a decrease in the response of the sugar neuron as a function of the duration of stimulation with this mixture.

\section{Behavioral testing of flies}

Recently hatched Sarcophaga sp. (supplied by The University of Michigan Amphibian Facility supported by NSF Grant GB4677 to George Nace) were waxed to wooden sticks and fed $2.0 \mathrm{M}$ sucrose and water for at least 2 days before testing. About 100 flies were used. The method of stimulation was to present to the fly a small circle of filter paper saturated with the appropriate chemical. Paper 
soaked in water or a sucrose solution was always readily seized and actively manipulated by the legs, and often held as long as permitted by the experimenter. When given sucrose, hungry flies immediately lowered the proboscis to the filter paper and drank.

The flies were treated with $0 \cdot 03 \%, 0 \cdot 1 \%, 1 \%, 3 \%$, and $10 \%$ Gymnema extract and tested with $0.1 \mathrm{M}, 0.3 \mathrm{M}$ and $1.0 \mathrm{M}$ sucrose. Nine of the fifteen possible combinations of Gymnema extract and sucrose were tested, including 10\% Gymnema extract with all sucrose concentrations and all Gymnema concentrations with $0 \cdot 1 \mathrm{M}$ sucrose. At least four flies were tested with each Gymnema-sucrose pair.

The testing procedure consisted of five steps. First, the flies were allowed to drink water until satiated. Then, they were tested for sucrose receptivity by observing whether lowering of the proboscis occurred. Drinking sucrose at this stage was not permitted. (Only those flies showing a positive response to sucrose were tested further.) Third, the flies were fully immersed in Gymnema extract for 1-2 min (as long as $6 \mathrm{~min}$ was tested). In about half of the tests the flies were not continuously immersed but were dipped in and out of the extract. Fourth, the flies were quickly rinsed with water or simply blotted dry. Fifth, sucrose was offered, and the flies were allowed to drink.

Flies were also tested with three additional sugars using the same procedure that was followed with sucrose. Glucose at $0.3 \mathrm{M}$ and $0.5 \mathrm{M}, 0.3 \mathrm{M}$ fructose and $0.3 \mathrm{M}$ maltose were offered after the flies had been dipped in $1 \%$ Gymnema extract for 2 min. Only one sugar solution was tested per day.

In a control experiment sixteen flies were satiated with water, tested for responsiveness to sucrose, treated with Gymnema extract $(0.03 \%, 1 \%, 3 \%$ and $10 \%$ solutions) and then tested with water. This controlled for the possibility that Gymnema treatment somehow reactivated a drive for water, per se. A second control experiment was carried out to determine whether olfactory stimulation by sucrose was responsible for the drinking of sucrose after Gymnema extract. Ten water-satiated flies were placed above a warm 2.0 M sucrose solution for $15 \mathrm{sec}$ and ten other flies above warm water. The experimenter looked for proboscis extension. A third control experiment was run to determine whether the crude Gymnema extract was itself a preferred stimulus. Three concentrations of Gymnema extract $(0.3 \%, 1 \%$ and $3 \%)$ were presented to flies on the paper discs after the flies had been satiated with water, and in some cases also tested for responsiveness to $0.5 \mathrm{M}$ sucrose, but prevented from drinking the sucrose.

\section{Crayfish}

\section{RESULTS}

Immersion of crayfish in the Gymnema extract did not measurably alter the normal EKG pattern, and in no case could we show that exposure of all known chemoreceptive sites to the substance diminished the response to glucose. Control animals that had not been exposed to Gymnema extract showed an average bradycardia of $14.3 \mathrm{sec}$ when given the standard dose of $0.25 \mathrm{M}$ glucose. Those that 
were exposed to the extract first were equally responsive when given sugar, showing an average cardiac inhibition of $16 \mathrm{sec}$. There was, in addition, no indication of a change in latency of onset of the reflex as a result of exposure to Gymnema extract.
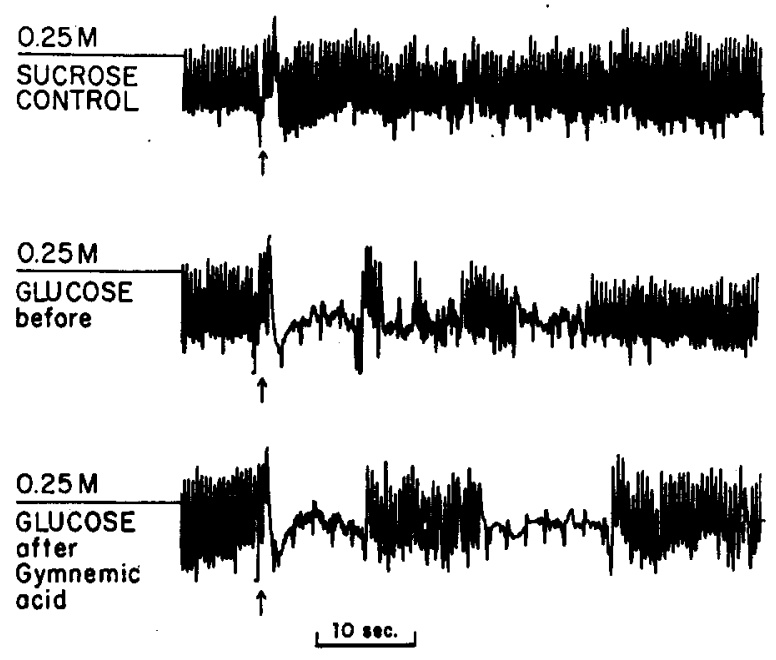

FIG. 1. EKG recordings from a crayfish obtained in response to the nonstimulating sugar, sucrose, and in response to glucose alone followed by glucose after Gymnema sylvestre. Interpretation of such records led to the conclusion that the Gymnema extract produces no effect on the glucose receptors of these animals.

A typical response of one individual to glucose alone and to Gymnema extract followed by glucose is shown in Fig. 1 .

Flies

It was not possible to detect an inhibitory influence of the Gymnema extract upon the impulse discharge of the sugar receptor. For example, the sugar receptor continued to respond actively to $0.5 \mathrm{M}$ sucrose for many minutes in the presence of $1 \%$ Gymnema extract (Fig. 2).

Forty-six out of fifty-two flies treated with the lower concentrations of Gymnema extract (i.e. $<10 \%$ Gymnema extract) gave positive behavioral responses to sucrose solutions. (A positive response was defined as full proboscis extension with or without sustained drinking. Six of the forty-six flies extended the proboscis but did not drink.) Twenty-six out of thirty-nine flies gave positive responses to sucrose with $10 \%$ Gymnema extract. The following results were obtained with three additional sugars after exposure to $1 \%$ Gymnema extract: four out of eight flies drank $0.3 \mathrm{M}$ glucose, six out of eight drank $0.5 \mathrm{M}$ glucose, six out of eight drank $0.3 \mathrm{M}$ fructose and eight out of eight drank $0.3 \mathrm{M}$ maltose.

In the control experiments only three of sixteen flies responded positively to water after Gymnema treatment. Definite proboscis extension occurred in only 
one of the ten water-satiated flies exposed to warm $2.0 \mathrm{M}$ sucrose vapor, and in none of the ten flies exposed to water vapor. There was no clear preference for Gymnema extract itself. One out of four flies responded to $0.3 \%$, five out of

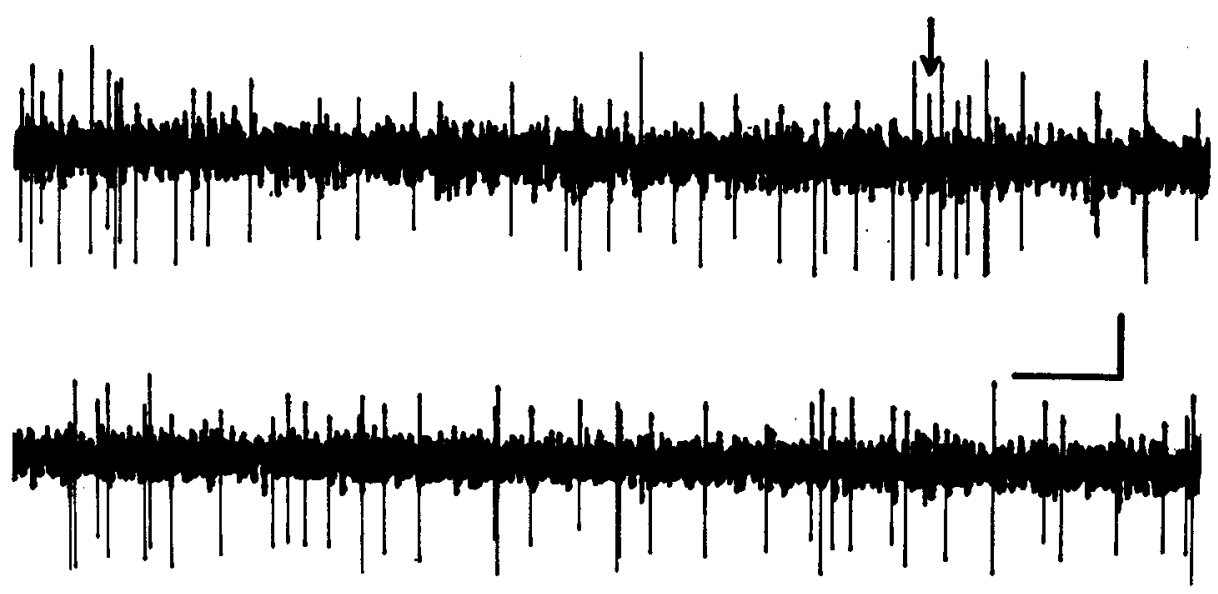

Fig. 2. Action potentials from a Sarcophaga labial hair, recorded through the stimulating pipette which contained a mixture of $0.5 \mathrm{M}$ sucrose, $0.05 \mathrm{M} \mathrm{NaCl}$ and $1 \%$ Gymnema extract. The top record was taken after $45 \mathrm{sec}$ of continuous application of the mixture to the hair tip; the bottom record after $8 \mathrm{~min}$ of the same application. About $75 \%$ of the spikes in each record are from the sugar receptor and $25 \%$ from the salt receptor. Thus, Gymnema extract was unable to prevent the sugar receptor from firing even after many minutes of application. The arrow points to a sugar receptor spike between two larger spikes from the salt receptor. Horizontal calibration mark: $0.5 \mathrm{sec}$. Vertical: $50 \mu \mathrm{V}$.

seventeen responded to $1 \%$ and none out of four responded to $3 \%$ Gymnema extract. Only two of all the responding flies actually drank.

\section{DISCUSSION}

Exposure to a wide range of Gymnema extract concentrations for several minutes failed to prevent the positive behavioral response to sucrose in flies. Control experiments indicated that the response to various sucrose concentrations was not a response to water, per se, nor was it a response to the odor of sucrose. Direct stimulation of the tarsal chemoreceptors with sucrose molecules in solution was required to elicit the feeding response. Though the crude $10 \%$ Gymnema extract is a black, viscous fluid, complete immersion in it prevented feeding in only $20 \%$ of the flies. It is likely that this inhibition was attributable to nonspecific trauma rather than to specific inhibition of the sugar receptor. It has been suggested that there are two types of active sites on the sugar receptor membrane -one sensitive to glucose and the other to fructose (Evans, 1963). The behavioral results with these two sugars suggest that Gymnema extract blocks neither site. The present electrophysiological measurements indicate that Gymnema extract 
does not have the potent inhibitory influence in Sarcophaga that it does upon the electrophysiological response to sugars in man and hamsters (Hagstrom, quoted by Pfaffmann, 1959; Diamant et al., 1965).

The major conclusion of these experiments is that a known inhibitor of sugar chemoreception in man is totally ineffective in blocking the response in the two invertebrates tested. The membrane binding sites for the common stimulating sugars must therefore differ in man and in these invertebrates, if one assumes a model similar to that proposed by Beidler (1954) to be operable in both cases. The crayfish receptors are primarily sensitive to those molecules having a free- $\mathrm{OH}$ on $\mathrm{C}_{1}$ such as glucose and xylose, while compounds having a glycosidic link at $\mathrm{C}_{1}$ such as sucrose are almost totally non-stimulating (Ashby \& Larimer, 1965). If the mode of operation of the active principle of Gymnema sylvestre is in fact one of competition with the normal stimulating molecule for sites on the receptor membrane (Warren \& Pfaffmann, 1959), one might conclude that it is not sufficiently active to block the highly specific glucose receptor of the crayfish.

The failure of the extract in blocking the response to sugars in Sarcophaga is also likely to be explained by the high specificity of the receptor sites. In the blowfly, Phormia, only a few carbohydrates are effective stimulants (Hodgson, 1957; Dethier, 1963). The present evidence points to the importance of the $\alpha$-D-glucopyranoside link in disaccharides and the $\mathrm{C}_{3}$ and $\mathrm{C}_{4}$ hydroxyl groups in hexoses (Evans, 1963).

The high degree of structural and probably conformational specificity in the fly and crayfish sugar receptors suggests that the active Gymnema molecule is unable to approach or combine effectively with the receptor membrane, and thus is unable to inhibit the receptor. It should not be concluded that the present experiment indicates a basic distinction between all invertebrate and vertebrate sugar receptors since there is some evidence that the albino laboratory rat is also immune to the differential inhibitory effects of Gymnema preparations (Oakley, unpublished observations).

Acknowledgements-We thank Mr. Gary Shelton, Miss Sibylle Herrmann, and Miss Toni Hall for technical assistance. The support of one of us (J. L. L.) by the Guggenheim Foundation is gratefully acknowledged.

\section{REFERENCES}

AshBY E. A. \& LARIMER J. L. (1965) Modification of cardiac and respiratory rhythms in crayfish following carbohydrate chemoreception. F. cell. comp. Physiol. 65, 373-379.

BeIDLER L. M. (1954) A theory of taste stimulation. Y. gen. Physiol. 38, 133-139.

DetrikR V. G. (1963) The Physiology of Insect Senses, Ch. 5, pp. 112-155. Methuen, London.

Diamant H., Oakley B., Strom L., Wells C. \& Zotterman Y. (1965) A comparison of neural and psychophysical responses to taste stimuli in man. Acta physiol. scand. 64, 67-74.

Evans D. R. (1963) Chemical structure and stimulation by carbohydrates. In Olfaction and Taste (Edited by Zotrerman Y.) pp. 165-176. Pergamon Press, New York. 
Hagstrom E. C. (1957) cited in Prafrmann C. (1959) The sense of taste. In Handbook of Physiology (Edited by FirLd J.) Section 1: Neurophysiology. Vol. I. American Physiological Society, Washington, D.C.

Hodgson E. S. (1957) Electrophysiological studies of arthropod chemoreception-II. Responses of labellar chemoreceptors of the blowfly to stimulation by carbohydrates. F. Insect Physiol. 1, 240-247.

HodGson E. S., LETTVIN J. Y. \& RoEDER K. D. (1955) The physiology of a primary chemoreceptor unit. Science, N.Y. 122, 417-418.

LARIMRR J. L. (1964) Sensory-induced modification of ventilation and heart rate in crayfish. Comp. Biochem. Physiol. 12, 25-36.

Warren R. \& PFaffmann C. (1959) Suppression of sweet sensitivity by potassium gymnemate. F. appl. Physiol. 14, 40-42. 